Brit. J. industr. Med., 1951, 8, 209.

\title{
THE BEARING OF EXPERIMENTAL PSYCHOLOGY UPON HUMAN SKILLED PERFORMANCE
}

\author{
BY \\ SIR FREDERIC BARTLETT \\ From the Psychological Laboratory, University of Cambridge
}

I am going to try to tell a story of interplay between ideas and experiments. The last 15 years have been a period of intense and concentrated activity for all psychologists working in the University of Cambridge. To those of us who have been closely identified 'with it, the whole development has assumed a character of exciting and engrossing adventure. I believe nobody, at the beginning, could possibly have foreseen to what precise issues our early, and very general, directing ideas would lead us. But as experiment has grown from experiment these ideas have become clarified and defined until we have now, so it seems to me and to many others, an approach and a technique in the controlled study of realistic human behaviour, which are of great significance in many fields, and certainly not least in the vast field of industrial medicine.

The volume and detail of work completed have been very great. If we consider the activities of only one of the Cambridge Units, the Unit for Research in Applied Psychology, established by the Medical Research Council in 1944, we find that up to July of the present year 156 reports, the large majority of which are directly experimental in character, have been circulated. To these must be added a considerable number of published papers, all similarly of a basic experimental character, which were issued by the Nuffield Unit for Research in Ageing, established two years later. Generous support from the Rockefeller Trustees, from all three of the Fighting Services of this country, and from a number of industrial sources, has produced its full share to swell the stream of recorded research. All the time there has been a steady growth of contributions from members of the University staff and from research students who have come to us from many parts of the world.
Since the bulk of this work is experimental, its detailed description has often taken a somewhat technical form. It would be unreasonable to expect any student or investigator, who has not himself been at the centre of operations, to be able, without great labour, to construct a clearly defined idea of what has already been achieved, and of the main growing points of present and future advance.

The time appears to have come for an attempt to present a synthetic view of the whole of this research, and to try to do it in a manner sufficiently free from technicality to render it possible for any interested reader to understand, and, if it seems desirable, to make use of the fundamental ideas which have shaped its development. This, therefore, is what I shall now try to do, and if I adopt a somewhat personal style, it is only because that seems the best way to convey a little of the thrill and feeling that something yet more important may be waiting "just round the corner", a feeling which all of us who have been actively concerned have had and still cherish.

\section{The Beginning}

It is always difficult to fix a beginning for any ideas which grow slowly into a new approach to longstanding problems and a new set of techniques for their study. No doubt in this case there was a process of gradually accumulating discontent with the classical methods of the experimental psychologists in a good many different areas of investigation. Visual perception had been dominated by the employment of squares, triangles, circles, and similar figures drawn in two-dimensional form on blank cards, very different in their properties and perceptual demands from the contours, shapes, and meaningful objects of the visual world, and usually with all the vitally important distance and depth 
characters lacking. In the early 1930s I myself had reached and published a demonstration that in memory studies great preoccupation with " nonsense " material may become more of a hindrance than a help. When, in the course of the second world war we were forced, for practical reasons, to ascertain how people use their skill, both mental and bodily, to track targets, to drive vehicles, to pilot airplanes, or to control them from the ground in concerted flight, we confronted much the same difficulty. The units used in the classical experiments, (simple thresholds of movement; reaction-times having, often enough, succession, but nothing that could be called an ordered sequence ; items in the skill separated from everything else, having the accredited form of "stimulus-response", but devoid of any character of accomplishment of an internally varied task) seemed, if they were forced into the world of everyday work and play, to have the same taint of " nonsense".

One day, fairly early on in the war, I had been out with Kenneth Craik to look at some new anti-aircraft equipment. When we were being driven back to Cambridge we fell into discussion about how to investigate air pilot's fatigue. For some time I had been trying to think how the conventional laboratory procedure for the study of fatigue might be supplemented and perhaps improved. The current methods, based almost wholly upon the investigation of simple and relatively isolated muscular and mental activities, seemed to me so devised that practically only three types of result could be recorded accurately : the amount of overall deterioration of work, the occurrence of checks and spurts in work, and, if the activity were pushed to a certain distance, the final collapse of work. What we needed to know was precisely how skill, in long-continued exercise, may change and perhaps deteriorate. So I asked Kenneth whether he could design and build controls for an experimental cockpit. We should, it seemed to me, try to pick out the " key ", or essential, elements in the control behaviour of a pilot flying by instruments. We should record these accurately, if need be for long periods, and also as much as possible of the associated but perhaps less critical activities. Then we should know, not only whether less or more work was being done, but also by what kinds and amounts of change in the co-ordinated control behaviour these, and other, variations, were being brought about.

As anybody who knew him will remember, Craik had a wonderful big black wallet. It was always stuffed to overflowing with odds and ends of bits of paper, with jotted notes in a most illegible handwriting about projected experiments, with dates of engagements seemingly in a terrific muddle, with impromptu drawings of experimental apparatus. Out came this wallet. He found an available bit of blank space. A diagram began to grow.

In a quite definite sense this was the beginning. The very next day Craik was in the laboratory workshop fashioning the experimental controls for what came to be known as "the Cambridge cockpit". The experiments were done later, first by Craik himself, and then, more fully, by G. C. Drew (now Professor of Psychology in the University of Bristol), and by Russell Davis (now Reader in Clinical Psychology in the University of Cambridge).

It is fair to claim that these experiments, more than any others, gave us the guiding principles which we have been able to use, in many applications, with growing definiteness, ever since. They demonstrated that the characteristic changes which take place in skilled human performance with long continued exercise are very different from those which normally mark the continuation by repetition of isolated muscular or mental activity. What made the demonstration possible was that we had refused to break up the skill into bits and pieces, and to be content with a measure of how well the performer could, at any stage, carry out a selected item of his work. We had discarded the view that if we could find out, in terms of time taken,- or effort expended, or degree of accuracy achieved, the normal limits of response for each successive move in the skill cycle, we could somehow infer what should be expected in terms of overall time, or effort, or accuracy for the whole cycle. We knew now that in order to discover anything important about skilled performance we must find a way to get right inside the skill itself, and to relate, within the experiment, actual overall measures with actual measures of the ways in which the internal constituents were being carried out.

It also became plain that, certainly in this type of skilled performance, and perhaps in others, not all of the internal constituents are equally important. There are "key" or " master" elements round which all others tend to be grouped, so that if the key activities are properly timed and carried out, all the rest will normally go well without much, if any, voluntary effort. How to discover the " master" constituents was obviously a question of predominant importance.

Moreover we learned that the absolute time (especially the least time) taken to complete any movement, or decision, involved in skilled activity, is of very little significance. What matters is the " spacing " or " timing" of movements, or decisions, or both, in their natural succession. This meant 
that "reaction-times", in the classical sense, could tell us very little, and we began to see why, in spite of the immense amount of work that had been done about them, not much had been learned from them that seems to have a practical or theoretical bearing upon the carrying out of any known skill. This " timing" obviously demanded consideration of the arrangement and interpretation of the perceptual " display", as well as of the consequent execution of any active movements. The " receptor" elements in skill, much neglected in many forms of motion study, at once assumed most important functions.

Finally an extremely interesting point emerged from these early experiments resting on the difference between what a man can do and what he will do. At every stage of a skilled performance, except at the very beginning, and not always then, two thresholds are measurable : the threshold of ability and the threshold of range, or of indifference. The first is the least perceptible change of display which can produce a response ; the second is the range of perceptible change beyond which a response will be made. The first can remain constant while the second has altered notably. On the face of it, it seemed that this must bring into our experiments the persistent and little understood condition called "motivation". But this was not motivation in any ordinary sense, for both to himself and to anybody else the performer could all the time remain as keen as ever. Some pilots, after about two and a half hours of continuous exercise, required instrument changes double or treble the size of those which were effective at the beginning, before they would do anything about them. Yet it was easily demonstrable that their ability threshold had not risen, and they were themselves entirely unaware of any difference in this aspect of their behaviour, and honestly claimed that they were doing as well as ever.

We had now, it appeared, achieved a promising method for experimental development, and we were faced by a number of intriguing but reasonably well defined questions. Obviously we must exploit in all possible ways the study and measurement of skilled performance in as natural a setting as possible, and in the form, not of analysed or broken up bits of the complex activity, but of continued exercise. We must try to find out how, in such continued exercise, "key" elements, or moves, can be identified. We must clear up the implications and conditions of spacing in display, and of timing in control. We must go much further in understanding the vitally important difference between what people can do and what they will do. We must be prepared to defend the view that, certainly for a long time, possibly for always, at various growing points our authority must be the trained judgment, what the medical man might call "diagnostic flair", and that though interpretations must be given as fully warrantable an experimental basis as possible, the experiments must be designed and developed not only with technical skill but also with psychological wisdom.

\section{Development}

In attempting to present a picture of the post-war developments of the experimental psychology of skill in Cambridge, I am bound to select only a few of what $I$ judge to have been crucial points of experimentation and interpretation. In fact every member of the working units and groups concerned has contributed towards whatever success has been won. Those whose work I have selected would be the first to agree that a very different selection might just as well have been made.

Importance of Display. - " Display " is the term which is used for all the environmental signals which the skilled performer uses to guide his work, to initiate and stop the movements or decisions which he has to make. "Control" is the term for the mechanical and electrical devices which have to be used by the performer in the vast majority of everyday skills, and also for the directing functions of decisions and movements themselves. It is clear that a part of the information which the operator is using comes from the display, in the sense just defined, and a part from the proprioceptive impulses set up by his movements. Perhaps there is also some direct effect of the central activity involved in making decisions.

It appears more and more certain that if there is one outstanding characteristic of efficient skilled performance, whether in work or in play, it is the consistent timing of successive steps or moves. We can show, for example, that the highly skilled transport driver tends to maintain a persistent pattern of behaviour, making the necessary adjustments of acceleration, deceleration, gear-changing, change and speed of change of direction, within small limits of difference relative to the overall rate at which he is travelling and to the rate of movement of other relevant items in his visual field. Neither to observation nor in measurement are the particular adjustments made at an enhanced speed. In fact, the skilled performer everywhere usually seems to have "plenty of time". The great thing is that successive moves flow one into another without check or hurry, without waiting or snatching. What sort of information is it that makes this possible, and does the information act all the time or only intermittently? 
G. C. Grindley and a number of collaborators have carried out a long series of experiments with very simple movements and pressures, by the exertion of which the operator is required to bring a pointer on a visual scale to a given position and stop it or keep it there. Any normal subject can learn this quickly, and provided the visual check is provided, can repeat or maintain the movement or pressure with great success. But as soon as the visual information is removed relatively large errors appear. They are, within limits, haphazard, and the subject himself knows little or nothing about them. With small movements and pressures the errors are predominantly of overshooting, but in whatever direction they start, they are apt to continue up to a limit and then be reversed. It seems as if the "stop" signal must come mainly from the display, and as if the proprioceptive information that is available operates only cumulatively and in an intermittent manner.

Normal skilled adjustments, however, do not consist of single directional movements. The moves form series, with varied change of speed, extent, and direction. Accordingly other investigators have carried out the same pattern of experiments, but with continuous tracking. The critical points are near by where marked changes are required, especially, in this case, changes in the direction of movement. If the display is removed, or made intermittent, the changes will normally be made too soon or too late-far more often too late-with consequent unwanted deceleration or acceleration afterwards.

The skilled operator is not using equally, or in the same manner, all the information that is available to him. In particular for stopping and starting, for deceleration and acceleration, and for their commonly associated changes of direction, display features, appreciated by exteroceptive perception, are of predominant importance, and make possible genuinely graded, as distinct from intermittently cumulative, responses.

Yet it is clear that the proprioceptive information is not being entirely ignored. C. B. Gibbs and others, for example, have shown that for many purposes in which successive positional adjustments are required, a pressure type of control, in which free movement is reduced to a minimum, is easier both to learn and to maintain even when display features are unchanged. And, for reasons at present unknown, this is particularly marked in relaxation phases of control, as contrasted with active contraction phases.

The broad conclusion from all these experiments is that display has a preponderatingly important part to play in all skilled operations at all of their stages. Where the operations are machine controlled, as is now the case with the vast majority of industrial skills, display design is from first to last, a matter of vital importance. Obviously no general discussion can deal wtth design in detail, though the details have been worked out in many instances. The important characteristics of display signals are : number, location, size, shape, direction and rate of change, and modal variety. In all of these the desirable feature is the greatest possible freedom from ambiguity. That display may possess this marked significance has a number of disturbing implications for many developments of motion study and job analysis.

Anticipation.-Many people, looking at quickmoving industrial operations, must have noticed that the operators seem to be interpreting signals ahead of the action point which they have reached. Yet hardly any investigator has paid much attention to the anticipatory functions which can be easily identified in almost all skilled performance. Innumerable studies and speculations have been made of and about remembering. But nobody until lately has seriously set himself to discover the normal range of anticipation, what it does to the movements and decisions that make up a skill, and through what mechanisms it operates. E. C. Poulton, H. Kay, Miss M. A. Vince, J. Leonard, and others decided to try to fill up the gap.

First they have shown that when display signals succeed one another fairly rapidly, especially in most psychophysical skills when the intervals are rather less than $0.5 \mathrm{sec}$., the interpreting senses do normally run ahead of their associated actions. This does not usually speed up the movements, but they succeed one another in a more fluent manner, and the resting interval from move to move is reduced, while at the same time the effort of changing, for example from one direction to another, is lessened.

There are at least three different kinds of anticipation. When signals can be treated as coming along in a single related stream, and each signal has its appropriate response, as in many conveyor belt operations, there is straightforward " receptor effector" anticipation. Its temporal limits are small and sharply cut off. It can work one, two, or, exceptionally, three steps ahead of the action point reached. It breaks down badly if the spacing of signals demands a more extended span, and it cannot deal with simultaneous signal streams all of which demand action.

But in many skills, both inside and outside industry, the signals possess an order and are more or less regularly recurrent. It is a character of 
human perception, as distinct from direct sensorial response, to be able to appreciate order, arrangement, and design. So the order and recurrence which have been perceived are used to prepare for their next appearance, and signal arrangements which have, in fact, already passed, are, so to speak, able to project action into the near future. Anticipation span is now extended to cover the whole of an order that can be assimilated by perception within whatever limits of time have been imposed by the manner of its presentation. The cost of this is obviously a loss of flexibility, for if anything novel breaks into the prospective signal scheme the skill suffers very seriously. Also there are severe limits to the number of overlapping, or concurrent, signal streams that can be dealt with by this type of anticipatory behaviour.

Suppose, however, that a situation has to be handled which is developing in many different directions at the same time. This is the kind of case that all centralized traffic control must deal with. It also recurs whenever reports are coming in from many different sources and have to be filtered, analysed, and passed on in a form suitable as a basis for quick decisions and action. It involves executive skill operating at a high level over a wide and varied area. Dr. N. H. Mackworth has been able to show that if, in such instances, it is possible, as it often is, to give foreknowledge of when and where critical developments are likely to occur, the normal result is that whatever the precise character of the critical developments may be, when they are reached, they will be dealt with more quickly, with less effort, and with improved accuracy. The exact limits of range of anticipation in this case depend upon the amount and nature of current activity, but the range is certainly vastly extended and flexibility is regained.

There is much yet to be discovered about the fundamental theoretical implications and the mechanism of these types of anticipatory behaviour, but once again it has been demonstrated that the accurate and sustained timing which is paramount in all efficient and economical skill depends both upon the nature, extent, and direction of the movements and decisions involved and upon a planned and adjusted spacing of display signals.

Speed and Load.- It has become more and more plain that the two predominant internal objective conditions of efficient skilled behaviour are speed and load. Both must be defined carefully. Speed is not measured adequately by the popular reactiontime technique. There is the time taken to initiate an action, once the signal has been given ; there is the time taken to complete the action; there is the "change over", or resting time, before the next required adjustment can be undertaken. It is certain that the first two have absolute limits, but these are very rarely approached in any conditions normally met with in industry or in everyday life. It is the " change over" time that is rather readily modifiable, and if an overall required speed of performance, combined with special conditions of display and control, either reduces or increases the change over time beyond limits, the whole performance will quickly lose its smooth and regular appearance. There is some evidence that most people are more intolerant to marked increase in change over, or resting time, than marked decrease. If this is really the case, the reasons, which are not yet known, must be of great interest. It is, however, quite certain that overall speeding-up of performance can alter the timing relations of the successive phases of serial response. It seems likely that it does this principally by a forced reduction of change over time, and when this passes a certain limit, the performance becomes ragged and uneven, and if it has to be continued the performer will inevitably suffer from fatigue and strain.

In whatever detailed manner it produces its effects, overall speed must be regarded as an independent condition of skilled performance. Moreover the present evidence is that if the overall speed is increased or decreased beyond the limits that are consistent with smoothly timed succession of response, the adverse effect piles up at a disproportionately rapid rate with every further increase or decrease.

Load depends on the number and arrangement of distinguishable signals, or signal sources, which have to be dealt with simultaneously or in very rapid succession. Load and speed may obviously be made to vary independently or in any desired order of dependence. Mackworth and Conrad have shown that while each produces its characteristic results, they can be readily combined, and then if either of them is increased or decreased, or if both are increased or decreased, beyond tolerable limits, the deterioration of performance that is produced is extremely marked. The common practice, which is to assume that if either is changed by a known proportionate amount, in order to maintain undiminished skill, the other must be changed in the opposite direction by a corresponding proportionate amount, is wrong. Further, load, in the sense of number of signals or signal streams, must be distinguished from load in the sense of arrangement of signals or signal streams. Some of the war-time experiments, based upon bomb aiming guided by signal clusters, showed that "outlier" signals are extremely likely to produce preponderant and 
exaggerated effects, and this has been confirmed with an experimental setting simulating machineminding. But at the same time the outlier signals are the ones most likely to suffer from the opposite error of intermittent or omitted responses in eontinued exercise.

Ceiling.-Both speed and load are, in a way, characteristics inherent in the skilled performance itself. There are also external conditions, which do not themselves work into the actual behaviour, but can affect it. The most important of these are the attendant environmental conditions : temperature ; humidity; the objective length of working spell ; the amount of overall bodily activity required; comfort. It was Mackworth who first demonstrated that these operate, in a normal working group, within fairly well defined and relatively constant "tolerance limits". Vary them within these limits and there is no significant change in the skill. Vary them even a little beyond the limits and the change is very striking. It seems exceedingly likely that the best way of defining " normality", whether in an individual or in a working team, in relation to any specified kind of skilled activity, may be in terms of "tolerance limits"; but more experiments are needed to demonstrate this. Whether there are any regular conditions which can be safely used to extend the ceiling, except for short intervals, is not yet known. The common practice is to offer enhanced rewards; but it is reasonably certain that this is ineffective except for spurt demands.

Key Moves.-Earlier in this article I said that one of our aims was to try to find out how key elements, or moves, in skilled performance could be identified. General master or key features, important in large classes of skilled activities, are not, as a rule, difficult to find. Even if, as often happens, they are, to begin with, reached by intelligent guessing, they can be experimentally tested. In fact all the characteristics of display and control and their exploitation that have been emphasized in this article are key features in the general sense.

In much the same manner it is easy enough to show that in psycho-motor skills the key characters occur predominantly where change of rate and extent, and more than anything else, of direction of movement are required. Active posture, out of which specific movements may flow easily, belongs to the same general master feature group. But so far nobody seems to know much about the postural conditions of efficient skill, except in very early stages of psycho-physical development, and in a general sense. There are more than enough opinions, but hardly any well-founded knowledge.
The topic waits for the sufficiently informed and ingenious experimenter.

The outstanding character of mental skills is that, compared with the bodily kind, they deal with situations at a distance, and therefore in symbols. But they have the same feature of moving in ordered and interrelated succession to whatever issue forms an accepted terminus.

And it is quite certain that in such moves there are key times and places, and if these can be picked out beforehand, so that the performer is ready to deal with them, all the near-by associated moves become relatively easy, uniform, certain, and fluent. In specific instances it is often possible to demonstrate by experiment such things as how far ahead it is worth while attempting to pick out and indicate these critical times and places, and in detail how, when this is done, the skilled performance becomes smoother, less tiring, and more effective. Mackworth has achieved this for the typical skill of traffic control, and he has shown in what ways and to what degree the whole process depends upon foresight. There is no doubt at all that it can be done in other instances as well. But when we ask how to detect, and especially how to impart this "gift" of identifying crucial times and places prospectively, at present we have no sure answer. This is one of the instances in which we must be willing to fall back upon " diagnostic flair".

It must be admitted that the same is true of particular key elements and combinations in a very large number of the skilled activities of daily life, especially when, as in most sports and games, the display has to be selected from a freely varying external environment by the performer himself. The general key features may tell us where to search, but variations of body build and mental endowment and acquisitions go heavily against the assumption of the single master-key.

Incentive and Motivation.-Motive is anything belonging to the performer himself which puts and keeps his specific skill processes in action. Incentive is anything coming to the performer from outside which sets some motive at work. The level and the persistency of skill depend on them both.

Little is known about either, and, as usual, the less is known the more seems to be asserted in speech and in writing. By far the commonest incentives used are a large variety of rewards and punishments. We know that the former are usually, except in special cases, more effective than the latter. We know something about the conditions under which the latter are of use. We know also that incentive, coming from the outside, operates with severe temporal limits, and can be 
held to something like a constant effect only by periodic increase. We know that whenever skilled work or play is being exercised at near tolerance limits, incentive will, and can, do nothing except in a short-lived spurt. Near the beginning of all of our work, Craik, pondering over operations of selfregulating machines, such as the velodyne motor, began to wonder whether it might be useful to think of motive as a kind of feed-back, operating from within a skill and currently with its unfolding, and acting as a kind of centrally appreciated standard, marking any difference between actual achievement and whatever it is the organism is set to achieve. And indeed this has proved to be a fruitful notion, directing many experiments ; and as I have indicated earlier, we have gone some way along this line, learning a little about the priorities and effectiveness of information given either intermittently, in a form of " knowledge of results", or currently, with practice of a skilled performance itself, through associated internal peripheral and central changes. But it is all the merest beginning, and in any case it cannot be the whole story. The real puzzle lies not in the relationship of performance and " set ", but in what settles and maintains the "set" itself.

\section{The Application of Experimental Techniques to Problems of Ageing}

When the Cambridge Nuffield Unit for Research into Problems of Ageing was formed, much of our early work on the measurement of skill had already been completed. There was at that time an imposing array of experimental work on the characteristic deteriorations of reaction which accompany increasing age. Nearly all of it was based upon studies of isolated responses; reaction-times; acuity thresholds of many kinds ; steadiness tests ; intelligence tests of the unit item variety ; problem solving; and the like. Where spells of working activity were studied, little attempt had been made to use any other measures except those of overall performance : amount produced, total errors, total times taken, amount of effort expended, and so on. The general picture obtained was one of small and negligible deterioration at relatively early age, followed sometimes by recovery and sometimes by slight persistent decline, and finally, at advanced age, very often a catastrophic fall.

It seemed to us that the time had come to approach the whole subject in a different manner. Because we knew most about these we resolved first to study the relatively light skilled operations which in fact, owing mainly to improved instrumental and machine technology in all directions, are coming to play a more and more dominant part in organized industry. Our skills must be allowed to keep their natural order of expression, and our measures must go inside the skills. We must give the operative conditions of design in display and timing in control, of speed, load, and ceiling, our primary attention. We must not be too much hunted by the notion of deterioration of skill alone, and supposing that we did find that there are changes in the manner of skilled performance which are characteristic of broad age ranges, we must find out whether these have important implications in regard to the most economical learning of skill at different ages, and its continued, day to day exercise.

The programme was large and undeniably ambitious, but Mr. A. T. Welford and his associates have already shown beyond question that its broad guiding ideas are fruitful and practicable.

First there are a number of technical and technological points of method connected with measuring skilled work from the inside, and with relating whatever goes on inside the skill with a measure of what is produced, or with an overall performance score. Even when we have demonstrated that the measures that we most need are related measures of timing in control and spacing in display, if we are to remain loyal to our view that these must be sought within the natural skill sequence, and not once only, but in continued exercise, any ordinary methods of recording and evaluating are excessively difficult and time consuming. Welford has, however, been able to develop an electronic time-and-error recording device which gives us the critical scores we need in such a form that they can be fed into automatic computing machines to give us speedily whatever statistical analysis is desired. There seems every prospect that this method of recording and evaluation can be greatly extended, and that it will open up fields of investigation which have so far been too difficult to explore in any detail.

The general story is consistent with most of what has been said already. Laboratory and field studies alike show that, as age increases, it is the timing of control responses that suffers most. The difficulty begins to appear early, in the late twenties and early thirties, but except under severe speed and load stresses, it is usually countered by a more "canny" use of the display signals, especially, probably, by a more deliberate and effective, though usually unwitting, extension of anticipation, and often by some reduction in a number of movements and decisions. Consequently overall performance does not suffer, and may even go on improving, unless there are rather drastic changes in the general design of the methods and environmental conditions of the performance. The bother is that nowadays very rapid improvements in machine design make 
such drastic changes common, and unless these are introduced with care and wisdom, serious difficulties are likely to develop, especially in the age range from about 40 to about 60 .

The evidence also shows that the increased intolerance to speed which accompanies ageing is not, during the period of a normal working life, or even rather longer, due to any natural increase in the time taken to initiate a response, or to the movement time itself, but to irregularities of change-over time, particularly, probably, in rather long spells of continued activity. Field studies appear to show that the trouble is most acute when skilled movements have to be combined with a large amount of general bodily activity, and both the movements and the general activity have to be carried out under imposed speed stress.

What causes the greatest difficulty of all is dealing with novel clusters of signals, and especially from the forties onwards the skilled operator cannot reasonably be expected to make the necessary readjustments quickly and automatically. Whether a little judicious retraining could maintain the required level of performance is not yet certain. Very likely it could, but the method of training must certainly be adapted to the age-range of the performer. In a broad sense it seems to be coming out more and more that display and load assume greater importance as age increases.

Any attempt to explain the precise mechanism of all this is at present bound to be pretty much of a guess. But it seems not unlikely that as what is called " middle age" approaches, there is a tendency to make more and more exclusive use of the second of the three main types of anticipation. The appreciation of order and pattern in events requires certainly an alert intelligence. But it can, though of course it need not, avoid the added burden of a recurrent or continuously active selective observation. The anticipatory function in rapid skilled performance cannot be avoided, for without it the essential smooth quality of skill will be lost. It may be that there is normally some retraction of span or range of direct exploratory anticipation, and with it some extension of span of the kind that rests upon short or long-term storage or recall. There is then inevitable loss of flexibility.

In spite of all of this, skill prognosis for age becomes steadily more and not less favourable as we come to understand better the nature of the changes that take place. Given a due regard to essential psychological conditions, a normally healthy population should be able to maintain and modify acquired skills, and even achieve new ones, well beyond any age that is now considered to mark the term of an active working life.
We began all our work on ageing in the laboratory. This was partly because we were proposing to develop new methods of assessment, and partly because we knew that when we went out into the industrial field we must be equipped with at least some guiding ideas of what to look for. Without these, observation of the field type gets bogged in a mass of varied detail. But observation alone, and even observation and field experiment cannot carry us all the way when we are concerned with direct industrial problems. We must have records, especially when types of training and learning about which practically nothing is known come up for consideration, as they obviously do in this case. We have found that reliable and relevant industrial records bearing upon the problems which have been indicated are very rare indeed. It is a matter of great urgency that this gap should be filled.

\section{The Study of Human Skill, Performance, and Industrial Medicine}

No doubt it is right and natural that industrial medicine should still be greatly preoccupied with detailed studies of specific hazards which must be encountered in particular industries, groups of industries, and industrial operations; and with problems concerning the treatment of the worker who falls a victim to these hazards, and how they may be prevented or the workers adequately safeguarded. Toxicological problems, a large variety of types of specific industrial diseases and acquired disabilities of bodily function, the treatment and prevention of burns and other injuries-these and many other questions of the kind that everybody at once recognizes to be "straight medical", must remain in the forefront of the industrial medical officer's attention.

Yet nobody can now ignore the facts that modern large scale and highly organized industry has to face up to a lot of human problems, the criteria for the occurrence and severity of which are limited efficiency, the piling up of unwanted and avoidable fatigue and strain; an accumulation of errors leading to accidents that could just as well never occur at all; irregular working habits, and the growth of simmering or open dissatisfaction often leading to dispute, the real causation of which is frequently misunderstood. For the most part these maladjustments do not issue in any definite or identifiable bodily disease, but in extreme cases, which are nevertheless sufficiently common to lead to plenty of trouble, they do produce a crop of nervous and mental disorders.

In all these instances the basic conditions are exactly the same as those which produce the prob- 
lems which are generally regarded as a proper concern of an industrial medical service. The body and the mind are set to work under circumstances with which they cannot deal without impairment of the activity that is demanded. It is the medical habit of mind, of learning to identify early symptoms of such impairment, and then probing beneath the surface to find out their real causation, that is most of all needed. It should never be forgotten, though it often is, that the body that was responsible for nearly all the pioneer work in this country on the scientific investigation of the matters which I have been discussing, and for the great bulk of its subsequent development, was the Medical Research Council through its Industrial Health Research Board : and that very nearly all the other organizations which have played a great part in the same field during the last 15 years or so have also had extremely strong medical interests.

It has been one of my chief aims, in writing this article, to show that the study of skill in industry, and the general and specific conditions alike of its impairment and of its healthy exercise, has become as technical as that of any of the widely recognized industrial diseases and hazards. The time has come, I would maintain, when every progressive industrial medical service must have its own group of professionally qualified investigators well trained in the principles and practice of experimental psychology. Their relation to the practising industrial medical officer should be exactly that of the toxicologist, or of the specialist in chest or heart diseases. The doctor in the industrial field must know what to look for, what records he, more than anybody else in industry, is in a position to appreciate and can be relied upon to collect systematically and in a sensible form. Now that we are certain that skill can and must be studied in its natural setting of sequential exercise, now that we know something about the key features of its efficient practice, there is a vast amount of valuable information and original observation which can be collected and carried out without any elaborate instrumentation.

I should be the last person to maintain that the approach which I have here attempted to describe, is the only one that should be tried. By all means let influential people go on seeing what they can do by repeated exhortation. Give all possible chances to every wise development of the " science of human relations". But unless, also, the conditions affecting the healthy and productive exercise of skill are studied scientifically and secured in practice, any number of appeals, any amount of " understanding " management, and the most expensive development of external incentives and amenities in the factory, will not heal troubled bodies, troubled minds, and a troubled society. 